\title{
LINEAR INVERSE PROBLEMS FOR MARKOV PROCESSES AND THEIR REGULARISATION
}

\author{
UMUT ÇETIN
}

ABSTRACT. We study the solutions of the inverse problem

$$
g(z)=\int f(y) P_{T}(z, d y)
$$

for a given $g$, where $\left(P_{t}(\cdot, \cdot)\right)_{t \geq 0}$ is the transition function of a given symmetric Markov process, $X$, and $T$ is a fixed deterministic time, which is linked to the solutions of the ill-posed Cauchy problem

$$
u_{t}+A u=0, \quad u(0, \cdot)=g,
$$

where $A$ is the generator of $X$. A necessary and sufficient condition ensuring square integrable solutions is given. Moreover, a family of regularisations for above problems is suggested. We show in particular that these inverse problems have a solution when $X$ is replaced by $\xi X+(1-\xi) J$, where $\xi$ is a Bernoulli random variable and $J$ is a suitably constructed jump process. The probability of success for $\xi$ can be chosen arbitrarily close to 1 and thereby leading to a jump component whose jumps are rarely visible in the practical implementations of the regularisation.

\section{INTRODUCTION}

Suppose that $X$ is a Markov process taking values in some topological space, $\mathbf{E}$, and let $\left(P_{t}\right)_{t \geq 0}$ be a strongly continuous semigroup describing the movement of $X$ in its state space through time. Let us consider the following integral equation

$$
g(z)=\int f(y) P_{T}(z, d y)
$$

for a given $g$ and a fixed deterministic $T \geq 0$. Put differently, the above can be viewed as recovering an input signal, $f$, from a blurred output, $g$, which is corrupted by some noise described by the kernel $P_{T}$. This is an inverse problem which is present in many fields of science and technology. In image processing solving this inverse problem corresponds to the reconstruction of an image from the available data as in, e.g., tomography (see [3]). In statistics one is often interested in estimating the density function, $f$, of a certain random variable using the observations of a related one with density $g$, which is linked by some kernel $K$ to the original density via the equation $g=K f$. Vardi and Lee [24] show that such inverse problems can be interpreted as a statistical estimation problem from an incomplete data if it admits a positive solution. Under the assumption of the existence of a positive solution to $g=K f$ they develop

Date: November 12, 2019. 
a maximum likelihood (ML) algorithm to solve the estimation issue and apply their methodology to problems arising from optimal investment, emission tomography, and image reconstruction due to motion blurring. More recent works on the interplay between ML estimators and inverse problems with positivity constraints include [23], [12], and [19], and [6] is an excellent introduction to inverse problems in statistics and a survey of available methods. Note that the inverse problem given by $g=K f$, where $K$ is a non-negative bounded linear operator on a Hilbert space, can be recast in the form of (1.1). Indeed, supposing without loss of generality that the norm of $K$ is less than 1 , if we define the operator $A:=-\log K$ (consult the beginning of the next section or Chapter 1 of [17] for the construction of this operator), then $A$ will correspond to the infinitesimal generator of a Markov process whose transition function at time 1 coincides with $K$, i.e $g=K f$ becomes $g(z)=\int f(y) P_{1}(z, d y)$, where $\left(P_{t}\right)_{t \geq 0}$ is the semigroup of the Markov process with generator $-\log K$. Thus, the method that we shall describe below will contain as special cases many inverse problems in the literature and, in particular, the above density estimation problem of statistics. Moreover, the existing literature typically assumes that $K$ is a compact operator to arrive at a simple singular value decomposition. We will not need this assumption in what follows and thereby considerably extend the scope of the methodology for solving such inverse problems.

The inverse problem in (1.1) has an alternative partial differential equation (PDE) interpretation. Suppose that for a given $g$ and fixed $T>0$ one can find a solution, $f$, to (1.1). Then, one can easily show that $u(t, \cdot):=P_{T-t} f$ is a solution to the following:

$$
u_{t}+A u=0, \quad u(0, \cdot)=g,
$$

where $A$ is the generator of $X$. If $A$ is a differential operator, the above is a backward PDE with an initial condition ${ }^{1}$. Such equations are known to be ill-posed in the sense of Hadamard that either there exists no solution or the solution is non-unique, or the unique solution does not have a continuous dependence on the initial data, $g$. In Remark 3 we suggest a measure of ill-posedness for the inverse problems considered in this paper and show in particular that the degree of ill-posedness grows exponentially with $T$.

One motivation for the study of this particular problem is the Kyle model of financial economics (see [20] for the original formulation, [2] for the rigorous formulation in continuous time, and [10] for its various generalisations in the context of diffusion processes). The Kyle model is the canonical model of the market microstructure theory to study market equilibrium among heterogeneously informed agents. When the market makers in this model are risk-averse and the informed trader has a timestatic signal, Çetin and Danilova [9] have established the existence of equilibrium in

\footnotetext{
${ }^{1}$ We use the term backward to describe this PDE despite the fact that it is stated with an initial condition. Note that the equation $u_{t}+A u=0$ is well-posed if it is given with a terminal condition of the form $u(T, \cdot)=f$, in which case the terminology backward PDE becomes natural. Thus, our choice is motivated by the emphasis on the ill-posed nature of such equations when they are stated with an initial condition.
} 
this model by showing the existence of a solution for the following forward-backward system:

$$
\begin{aligned}
H_{t}+\frac{1}{2} H_{y y} & =0 \\
d Y_{t} & =d B_{t}-\frac{1}{2} \gamma Y_{t} H_{y}\left(t, Y_{t}\right) d t \\
H\left(1, Y_{1}\right) & \stackrel{d}{=} f(\eta),
\end{aligned}
$$

where $B$ is a Brownian motion, $\eta$ is a standard normal representing the static information of the informed trader, $f$ is a strictly increasing continuous function and $\stackrel{d}{=}$ stands for equality in distribution.

However, to better approach reality one must assume that the informed trader has a time-dynamic signal that converges to a standard normal random variable (after some normalisation) at $t=1$ as in [5]. Assuming her signal is a mean-zero Gaussian martingale $Z_{t}$ with $\mathbb{E}\left[Z_{t}^{2}\right]=V(t)>t$ for $t \in(0,1)$ (see [5] for the necessity and interpretation of this condition), the equilibrium considerations as in [5] or [10] show that (1.3) is not sufficient to establish an equilibrium and one also needs to solve for every $t<1$ and $y \in \mathbb{R}$ the following integral equation:

$$
g(z):=p\left(t, y ; 1, H^{-1}(1, f(z))\right) \frac{d}{d z} H^{-1}(1, f(z))=\int_{-\infty}^{\infty} q(1-V(t), z-x) f(x) d x,
$$

where $q(\cdot, \cdot)$ is the transition density of the standard Brownian motion and $p$ is the transition density of the process $Y$ in (1.3). Using the symmetry of $q$ the above integral equation is easily seen to be an example of $(1.1)$ when $\left(P_{t}\right)$ is the Brownian semigroup.

Inverse problems arising from (1.2) also become relevant when one is interested in the asymptotics of forward performance processes in Mathematical Finance Theory. Shkolnikov et al.[22] have recently studied this problem in incomplete markets and established its connection to a family of ill-posed Hamilton-Jacobi-Bellman equations.

In what follows we aim to find necessary and sufficient conditions for (1.1) to admit a suitably integrable solution. There do not seem to be many attempts in the literature to characterise the solutions of such an inversion. The only attempt when $\left(P_{t}\right)_{t \geq 0}$ is the transition function of a linear Brownian motion is by Widder. In [25] and some subsequent works Widder provides some necessary and sufficient conditions for the existence of a solution to this inverse problem, which he calls Weierstrass transform.

We show in Theorem 2.2 that (1.1) has a square integrable solution if and only if

$$
\int_{0}^{\infty} I_{0}(2 \sqrt{2 T t}) \int_{0}^{\infty} J_{0}(2 \sqrt{t s}) e^{-\alpha s}\left(P_{s} g, g\right) d s d t<\infty
$$

where $J_{0}$ (resp. $I_{0}$ ) is the (resp. modified) Bessel function of the first kind of order 0 . Additionally, the same theorem gives a formula for the inversion. Section 2 also contains a number of alternative criteria for the characterisation of the domain of 
$P_{t}^{-1}$. In particular it is observed that the finiteness of the double integral above can be recast in terms of the last passage times in the case of one-dimensional regular diffusions. Moreover, Corollary 2.4 gives us a numerical recipe by means of a Picard iteration to deduce the convergence of this integral.

As we mentioned earlier there is no solution to (1.1) in general for an arbitrary transition function. Theorem 3.1 introduces a family of regularisations of (1.1), which are essentially small perturbations of the original problem aimed at obtaining a solution for any given $g$. Moreover, the solution of the regularised problem is characterised in terms of the minimiser of an associated optimisation problem.

Corollary 3.1 gives a remarkable special class of regularisations suggested by Theorem 3.1. It shows that if we construct a new Markov process that amounts to choosing randomly between the original process, $X$, and a suitable pure jump process, then the inverse problem will have a solution for every $g$ as soon as $P_{T}$ is replaced by the corresponding transition function of the new Markov process. For example, when $X$ is a Brownian motion, the inverse problem can be turned into a well-posed one by replacing the Brownian motion with a mixture of a Brownian motion and a compound Poisson process whose jumps are normally distributed. Such mixtures of the original Markov process and a jump process are easy to construct and one can choose the probability of choosing the jump process arbitrarily small so that the jump component is virtually absent in implementations. This mixture also regularises the ill-posed backward PDE (1.2) by transforming it to a partial integro-differential equation using an arbitrarily small perturbation.

Although we are able to give a necessary and sufficient condition for the existence of a solution to (1.1), what is particularly missing in this paper is a comparison result. Namely, if we know that $P_{T}^{-1} h$ exists for some $h$, what kind of relationship between $g$ and $h$ would entail that $g$ is also in the domain of $P_{T}^{-1}$ ? A comparison theorem in the spirit of the ones that can be found in the literature on the Skorokhod embedding problem could be very useful. Falkner [16] has shown (under a duality assumption and another mild condition) for a general transient Markov process, $X$, with potential operator $U$ that if $U \mu \leq U \nu$ for measures $\mu$ and $\nu$, then one can find a stopping time $\tau$ such that $X_{\tau}$ has the law $\mu$ if $\nu$ is the distribution of $X_{0}$. Note that in order for $g$ to be in the domain of $P_{T}^{-1}$ it is necessary that $U g \leq U h$ for some $h$ in the domain of $P_{T}^{-1}$. However, the following counterexample ${ }^{2}$ shows that this necessary condition is not sufficient: let $g$ be the distribution of $X_{\tau}$, where $\tau=\inf \left\{t \geq T:\left|X_{t}\right|>a\right\}$ and $X$ is a standard Brownian motion with $X_{0}=0$ and killed as soon as it exits $(-2 a, 2 a)$. Clearly, $U g \leq U \mu$, where $\mu$ is the distribution of $X_{T}$. However, $g$ cannot be in the domain of $P_{T}^{-1}$ since it has a point mass.

The outline of the paper is as follows. Section 2 presents the setup and introduces the inverse problem. It contains Theorem 2.2 that gives the necessary and sufficient condition for the inversion along with the inversion formula. Section 3 is devoted to the regularisation of the inverse problem and includes in particular Corollary 3.1,

\footnotetext{
${ }^{2}$ This example is due to David Hobson.
} 
which states that the inverse problem has a solution when $X$ is replaced by a mixture of $X$ and a jump process.

\section{An INVERSE PROBlem FOR A SYMMETRIC MARKOV PROCESS}

Let us fix a Borel right Markov process $X=\left(\Omega, \mathcal{F}, \mathcal{F}_{t}, X_{t}, \theta_{t}, P^{x}\right)$ with lifetime $\zeta$, state space $(\mathbf{E}, \mathscr{E})$, sub-Markovian semigroup $\left(P_{t}\right)$, and resolvent $\left(U^{\alpha}\right)$. Suppose that $\mathbf{E}$ is a locally compact separable metric space and $\left(P_{t}\right)$ is $m$-symmetric with respect to a $\sigma$-finite measure $m$ on the Borel $\sigma$-algebra $\mathscr{E}$ with $\operatorname{supp}[m]=\mathbf{E}$. More precisely, we assume that $\left(P_{t}\right)$ can be extended to a strongly continuous sub-Markovian semigroup on $L^{2}(\mathbf{E}, m)$ such that

$$
\left(P_{t} f, g\right)=\left(f, P_{t} g\right), \quad \forall f, g \in L^{2}(m),
$$

where $(\cdot, \cdot)$ denotes the inner product with respect to $m$, i.e. $(f, g)=\int_{\mathbf{E}} f g d m$ for $f, g \in L^{2}(\mathbf{E}, m)$. We also assume that $(\mathbf{E}, \mathscr{E}, m)$ is a separable measure space, which in turn implies that $L^{2}(\mathbf{E}, m)$ is a separable Hilbert space. In the sequel we shall simply write $L^{2}(m)$ instead of $L^{2}(\mathbf{E}, m)$.

The generator $A$ of $\left(P_{t}\right)$ is defined as usual via

$$
\begin{aligned}
A f & =\lim _{t \rightarrow 0} \frac{P_{t} f-f}{t} \\
\mathscr{D}(A) & :=\left\{f \in L^{2}(m): \text { The limit }(2.4) \text { exists in } L^{2}(m)\right\} .
\end{aligned}
$$

Consequently $-A$ is a non-negative definite symmetric operator on $L^{2}(m)$. Thus, there exists a spectral family ${ }^{3}\left\{E_{\lambda}: 0 \leq \lambda<\infty\right\}$ of projection operators such that $-A=\int_{0}^{\infty} \lambda d E_{\lambda}$. This further entails

$$
\mathscr{D}(A)=\left\{f \in L^{2}(m): \int_{0}^{\infty} \lambda^{2} d\left(E_{\lambda} f, f\right)<\infty\right\} .
$$

Moreover, if $\phi: \mathbb{R}_{+} \rightarrow \mathbb{R}$ is a continuous function, $\phi(-A)$ is another symmetric operator on $L^{2}(m)$ with the spectral representation $\int_{0}^{\infty} \phi(\lambda) d E_{\lambda}$ and domain

$$
\mathscr{D}(\phi(-A))=\left\{f \in L^{2}(m): \int_{0}^{\infty} \phi(\lambda)^{2} d\left(E_{\lambda} f, f\right)<\infty\right\} .
$$

In particular, for each $t>0$ and $\alpha>0, P_{t}=\int_{0}^{\infty} e^{-\lambda t} d E_{\lambda}$ and $U^{\alpha}=\int_{0}^{\infty} \frac{1}{\lambda+\alpha} d E_{\lambda}$, and obviously, have the whole $L^{2}(m)$ as their domain. When $X$ is transient, the potential operator is also given by $U=\int_{0}^{\infty} \frac{1}{\lambda} d E_{\lambda}$. We refer the reader to Appendix A.4 of [7] for a brief account of the spectral theory associated with the generators of strongly continuous symmetric semigroups and the corresponding spectral calculus.

\footnotetext{
${ }^{3}$ That is, 1) $E_{\lambda} E_{\mu}=E_{\lambda}, \lambda \leq \mu$;2) $\lambda \mapsto E_{\lambda} f$ is right continuous for any $f \in L^{2}(m)$; and 3) $\lim _{\lambda \rightarrow \infty} E_{\lambda} f=f$ for all $f \in L^{2}(m)$. In particular $\left(E_{\lambda} f, g\right)$ is of bounded variation in $\lambda$ for any $f, g \in L^{2}(m)$.
} 
Example 2.1. Let $\mathbf{E}=(l, r)$ with $-\infty \leq l<r \leq \infty$ and consider a one-dimensional regular diffusion on natural scale defined by the generator

$$
A f=\frac{\frac{1}{2} d \frac{d f}{d x}-u d k}{d m},
$$

where the killing measure $k$ and the speed measure $m$ are Radon measures on $(\mathbf{E}, \mathscr{E})$. In the absolutely continuous case the generator becomes a differential operator:

$$
A f=\frac{\sigma^{2}}{2} f^{\prime \prime}-c f
$$

where $\sigma: \mathbf{E} \rightarrow \mathbb{R}_{++}$and $c: \mathbf{E}: \rightarrow \mathbb{R}_{+}$are measurable functions.

McKean [21] has shown that the transition function, $\left(P_{t}\right)$ possesses a symmetric density, $p$, with respect to $m$ such that

$$
p(t, x, y)=\int_{0}^{\infty} e^{-\lambda t} e(x, y, d \lambda)
$$

where $e(x, y, \cdot)$ is a measure on $[0, \infty)$ with $e(x, y \cdot)=e(y, x, \cdot)$. We refer the reader to [21] or [18] for more details on the general theory of one-dimensional diffusions and the eigendifferential expansions of their transition densities. Observe that the spectrum of the generator in general has a continuous part.

When the diffusion has no natural boundaries Elliott [14] has shown earlier that the spectrum of the generator is discrete, which in turn implies that the transition density with respect to $m$ is given by

$$
p(t, x, y)=\sum_{n=0}^{\infty} e^{-\lambda_{n} t} \phi_{n}(x) \phi_{n}(y),
$$

where $\left(\lambda_{n}\right)$ is an unbounded sequence of eigenvalues corresponding to the equation $A \phi_{n}=-\lambda_{n} \phi_{n}$ for appropriate boundary conditions given by the behaviour of the diffusion near $l$ and $r$, where $\phi_{n}$ is the corresponding eigenfunction.

Example 2.2. Suppose that $q(x, y)=q(y, x)$ and $q d m$ defines a transition function on $(\mathbf{E}, \mathscr{E})$. In particular,

$$
\int_{\mathbf{E}} q(x, y) m(d y) \leq 1
$$

Then, it can be directly verified that $A$ defined by

$$
A f(x)=\int_{\mathbf{E}} f(y) q(x, y) m(d y)-f(x)
$$

is a bounded symmetric operator corresponding to a Markov jump process (see, e.g., Section 4.2 in [15]) that remains constant between jumps of a Poisson process with unit intensity and moves between the states of $\mathbf{E}$ according to the kernel $q$, or is being sent to the cemetery state with probability $1-\int_{\mathbf{E}} q(x, y) m(d y)$. 
In the particular case of $\mathbf{E}=\mathbb{R}, q(x, y)=q(y-x)$ for some symmetric function, $q, A$ is the generator of a compound Poisson process whose jumps have a symmetric distribution around 0 with $m$-density $q$, provided

$$
\int_{-\infty}^{\infty} q(x, y) m(d y)=1, \forall x \in \mathbb{R}
$$

Next consider the inverse operator, $P_{t}^{-1}$, for $t>0$. That is, $g \in \mathscr{D}\left(P_{t}^{-1}\right)$ if $g \in$ $L^{2}(m)$ and there exists $f \in L^{2}(m)$ such that $P_{t} f=g$. In this case we shall define $P_{t}^{-1} g$ to be $f$. Note that this operation is well-defined. Indeed, if $f_{1}, f_{2} \in L^{2}(m)$ are such that $g=P_{t} f_{1}=P_{t} f_{2}$, then $P_{t}\left(f_{1}-f_{2}\right)=0$. However, in view of the spectral representation of $P_{t}$, this yields $\left(E_{\lambda}\left(f_{1}-f_{2}\right), f_{1}-f_{2}\right)=0$ for all $\lambda \geq 0$, which in turn implies $f_{1}=f_{2}, m$-a.e. due to the fact that $\lim _{\lambda \rightarrow \infty}\left(E_{\lambda} f, f\right)=(f, f)$ for any $f \in L^{2}(m)$.

Observe that, since $P_{T}$ is a bounded self-adjoint operator, $P_{T}^{-1}$ is also a symmetric operator on $L^{2}(m)$. The following, which should formally follow from spectral calculus, characterises $P_{T}^{-1}$ in terms of the spectral family $\left(E_{\lambda}\right)$.

Theorem 2.1. Let $P_{T}^{-1}$ be the inverse of $P_{T}$ for $T>0$. Then the following hold.

$$
\begin{aligned}
\mathscr{D}\left(P_{T}^{-1}\right) & =\left\{g \in L^{2}(m): \int_{0}^{\infty} e^{2 \lambda T} d\left(E_{\lambda} g, g\right)<\infty\right\} \\
P_{T}^{-1} g & =\int_{0}^{\infty} e^{\lambda T} d E_{\lambda} g .
\end{aligned}
$$

Proof. This is a direct consequence of the definition of $P_{T}^{-1}$ and spectral calculus (see Appendix A.4 in [7]).

Remark 1. Note that the condition for $g$ to be in the domain of $P_{T}^{-1}$ has strong consequence on the smoothness of $g$. For instance, if $X$ is a Brownina motion, $g$ has to be analytic to be in the domain of $P_{T}^{-1}$ for any $T>0$.

The above result illustrates the first difficulty with inverting $P_{T}$. When $A$ is an unbounded operator, which is usually the case, so is $P_{T}^{-1}$. In this case $P_{T}^{-1}$ will have a dense domain, the characterisation of which is one of the main goals of this paper.

On the other hand, if $A$ is bounded, $E_{\lambda}$ becomes the identity operator for all $\lambda \geq M$ for some $M<\infty$. In view of the above representation for $P_{t}^{-1}$ and its domain, this boundedness property will be inherited by $P_{t}^{-1}$.

Corollary 2.1. Suppose that the generator, $A$, of $\left(P_{t}\right)$ is bounded. Then $\mathscr{D}\left(P_{T}^{-1}\right)=$ $L^{2}(m)$ for each $T>0$. In particular (2.5) holds for all $g \in L^{2}(m)$.

Remark 2. It is tempting to conclude that $P_{T}^{-1} \mathrm{~g}$ is nonnegative when $g \geq 0$ and belongs to $\mathcal{D}\left(P_{T}^{-1}\right)$. This would be especially handy when one needs to estimate the true density $f$ by observing an auxiliary density $g$ using the relationship $g=P_{T} f$. However, the positivity of $f$ does not in general hold although one can find instances 
in the literature (see, e.g., the beginning of Section 3.3 in [19]) where this issue is overlooked.

To see this in a concrete example suppose that $X$ is an Ornstein-Uhlenbeck process, i.e

$$
X_{t}=X_{0}+B_{t}-r \int_{0}^{t} X_{s} d s, \quad r>0 .
$$

Then, conditional on $X_{0}=x, X_{t}$ is normally distributed with mean $x e^{r t}$ and variance $\frac{1-e^{-2 r t}}{2 r}$. The speed measure for this diffusion is given by

$$
m(d x)=e^{-r x^{2}} d x
$$

thus its generator, $A$, is symmetric with respect to $m$. Then, if one takes $g=x^{2}$, it follows from a simple computation that $g=P_{1} f$, where

$$
f(x)=e^{2 r} x^{2}-\frac{e^{2 r}-1}{2 r} .
$$

Note that both $f$ and $g$ belong to $L^{2}(m)$. However, $f$ is not always nonnegative on $\mathbb{R}$.

Remark 3. The inverse problem considered in this paper is typically severely ill-posed. To get an understanding of the degree of ill-posedness suppose that $A$ is unbounded and consider the sequence of operators $\left(B_{n}\right)_{n \geq 1}$ defined by

$$
B_{n}:=\int_{0}^{n} e^{\lambda T} d E_{\lambda}
$$

Note that each $B_{n}$ is a bounded operator and $B_{n} g$ converges to $P_{T}^{-1} g$ if $g \in \mathscr{D}\left(P_{T}^{-1}\right)$.

On the other hand,

$$
\sup _{g \in \mathscr{D}\left(P_{T}^{-1}\right)} \frac{\left\|B_{n} g-P_{T}^{-1} g\right\|^{2}}{\|g\|^{2}}=\sup _{g \in \mathscr{D}\left(P_{T}^{-1}\right)} \frac{\int_{n+}^{\infty} e^{2 \lambda T} d\left(E_{\lambda} g, g\right)}{\|g\|^{2}} \geq e^{2 n T}
$$

since if $g \in \mathscr{D}\left(P_{T}^{-1}\right)$, so is $g_{n}$, where

$$
g_{n}:=\int_{n+}^{\infty} d E_{\lambda} g .
$$

The above implies in a sense that the ill-posedness grows exponentially with $T$.

As mentioned in the Introduction the inverse problem (1.1) is intimately linked to the solution of a Cauchy problem, which becomes a backward partial differential equation when $A$ is a differential operator.

Corollary 2.2. The following hold for any fixed $T>0$.

(1) Suppose that $g \in \mathscr{D}\left(P_{T}^{-1}\right)$. Then there exist $(u(t, \cdot))_{t \in[0, T]}$ such that $u(t, \cdot) \in$ $L^{2}(m)$ for all $t \in[0, T]$, and

$$
u_{t}+A u=0, t>0, \text { and } u(0, \cdot)=g,
$$

where $u_{t}(t, \cdot):=\lim _{h \rightarrow 0} \frac{u(t+h, \cdot)-u(t, \cdot)}{h}$ and the limit is in $L^{2}(m)$. 
(2) Conversely, if there exists a family $(u(t, \cdot))_{t \in[0, T]} \subset L^{2}(m)$ solving (2.6) for a given $g \in L^{2}(m)$, then $g \in \mathscr{D}\left(P_{T}^{-1}\right)$.

Consequently, there exists a unique solution of (2.6) in $L^{2}(m)$ if and only if $g \in$ $\mathscr{D}\left(P_{T}^{-1}\right)$. Moreover, $P_{T}^{-1} g=u(T, \cdot)$.

Proof. Let $f=P_{T}^{-1} g$ and define $u(t, \cdot)=P_{T-t} f$. First observe that since $f \in L^{2}(m)$, $P_{t} f \in \mathscr{D}(A)$ for all $t>0$. Indeed,

$$
\int_{0}^{\infty} \lambda^{2} d\left(E_{\lambda} P_{t} f, P_{t} f\right)=\int_{0}^{\infty} \lambda^{2} e^{-2 \lambda t} d\left(E_{\lambda} f, f\right) \leq \frac{1}{t^{2} e^{2}} \int_{0}^{\infty} d\left(E_{\lambda} f, f\right)<\infty .
$$

Thus,

$$
A P_{T-t} f=-\int_{0}^{\infty} \lambda e^{-(T-t) \lambda} d E_{\lambda} f
$$

Moreover,

$$
\frac{d}{d t} P_{T-t} f=\frac{d}{d t} \int_{0}^{\infty} e^{-(T-t) \lambda} d E_{\lambda} f=\int_{0}^{\infty} \lambda e^{-(T-t) \lambda} d E_{\lambda} f
$$

by virtue of the dominated convergence theorem since $\left(E_{\lambda} f, f\right)$ is of bounded variation in $\lambda$ and $x^{2} e^{-2 x}$ is bounded on $[0, \infty)$. Therefore, $u$ solves $(2.6)$ since $u(0, \cdot)=P_{T} f=$ $g$.

Conversely, suppose $u$ is a solution of $(2.6)$ in $L^{2}(m)$. In particular, $u(t, \cdot) \in \mathscr{D}(A)$ for $t \in(0, T]$. Thus for any $t \in(0, T]$, we have

$$
u(t, \cdot)=g+\int_{0}^{t} u_{t}(s, \cdot) d s=g-\int_{0}^{s} d s A u(s, \cdot),
$$

where the integrals are to be understood as Bochner integrals in $L^{2}(m)$.

Next observe that for any $\lambda \geq 0$ and $f \in L^{2}(m)$,

$$
E_{\lambda} f=\int_{0}^{\lambda} d E_{\mu} f \in \mathscr{D}(A)
$$

and

$$
A E_{\lambda} f=-\int_{0}^{\lambda} \mu d E_{\mu} f
$$

Applying $E_{\lambda}$ to both sides of (2.7) and exploiting the commutativity of $E_{\lambda}$ and $A$ we obtain

$$
E_{\lambda} u(t, \cdot)=E_{\lambda} g+\int_{0}^{t} d s \int_{0}^{\lambda} \mu d E_{\mu} u(s, \cdot) .
$$

However, the unique solution of the above equation is given by

$$
E_{\lambda} u(t, \cdot)=\int_{0}^{\lambda} e^{\mu t} d E_{\mu} g
$$

which readily yields $d E_{\lambda} u(t, \cdot)=e^{\lambda t} d E_{\lambda} g$. Therefore,

$$
P_{T} u(T, \cdot)=\int_{0}^{\infty} e^{-\lambda T} d E_{\lambda} u(T, \cdot)=g .
$$


Since $u(T, \cdot) \in L^{2}(m)$, we deduce that $g \in \mathscr{D}\left(P_{T}^{-1}\right)$.

Thus, we have shown that there is a one-to-one correspondence between $\mathscr{D}\left(P_{T}^{-1}\right)$ and the $L^{2}$-solutions of (2.6). Moreover, since $P_{T}^{-1} g$ is uniquely determined, any solution of $(2.6)$ satisfies $u(T, \cdot)=P_{T}^{-1} g$.

Finally, by virtue of $d E_{\lambda} u(t, \cdot)=e^{\lambda t} d E_{\lambda} g$ we readily establish the uniqueness of $L^{2}$-solutions of (2.6) under the assumption that $g \in \mathscr{D}\left(P_{T}^{-1}\right)$.

Theorem 2.1 characterises the domain of $P_{T}^{-1}$ completely. However, it requires the knowledge of the spectral resolution. Theorem 2.2, on the other hand, determines the domain of $P_{T}^{-1}$ in terms of the transition function. Before its statement let us introduce a new operator on $L^{2}(m)$ :

$$
\mathcal{J}_{t}^{\alpha} g:=\int_{0}^{\infty} J_{0}(2 \sqrt{t s}) e^{-\alpha s} P_{s} g d s
$$

where $\alpha>0, J_{0}$ is the Bessel function of the first kind of order 0 , and the integral is to be understood as a Bochner integral. Since $J_{0}$ is bounded and $U^{\alpha}$ is a bounded operator, it follows that $\mathcal{J}_{t}^{\alpha}$ is also a bounded operator and, thus, has $L^{2}(m)$ as its domain.

Proposition 2.1. Let $\left(\mathcal{J}_{t}^{\alpha}\right)$ be the family of operators defined by (2.8). For each $t>0$ and $\alpha>0 \mathcal{J}^{\alpha}$ is a non-negative self-adjoint operator on $L^{2}(m)$ with the following spectral resolution:

$$
\mathcal{J}_{t}^{\alpha}=\int_{0}^{\infty} \frac{1}{\lambda+\alpha} e^{-\frac{t}{\lambda+\alpha}} d E_{\lambda} .
$$

Moreover, for any $g \in L^{2}(m)$, the mapping $t \mapsto\left(\mathcal{J}_{t}^{\alpha} g, g\right)$ is convex in $t$ and decreases to 0 as $t \rightarrow \infty$.

Proof. Let us first show that (2.9) holds. Indeed, using Fubini and the spectral representation of $P_{t}$ along with the fact that $\int_{0}^{\infty} e^{-\alpha s} J_{0}(2 \sqrt{t s}) d s=e^{-\frac{t}{\alpha}} / \alpha$ (see Table 29.2 in [1]), we obtain

$$
\left(\mathcal{J}_{t}^{\alpha} h, g\right)=\int_{0}^{\infty}\left(\int_{0}^{\infty} J_{0}(2 \sqrt{t s}) e^{-(\alpha+\lambda) s} d s\right) d\left(E_{\lambda} h, g\right)=\int_{0}^{\infty} \frac{1}{\lambda+\alpha} e^{-\frac{t}{\lambda+\alpha}} d\left(E_{\lambda} h, g\right),
$$

which yields (2.9). Thus,

$$
\left(\mathcal{J}_{t}^{\alpha} g, g\right)=\int_{0}^{\infty} \frac{1}{\lambda+\alpha} e^{-\frac{t}{\lambda+\alpha}} d\left(E_{\lambda} g, g\right) \geq 0
$$

since $E_{\lambda}$ is a non-negative operator. It can be checked directly that $\mathcal{J}_{t}^{\alpha}$ is symmetric, and therefore self-adjoint due to its boundedness.

The spectral representation also yields the monotonicity and the convexity of the map $t \mapsto\left(\mathcal{J}_{t}^{\alpha} g, g\right)$. The fact that $\lim _{t \rightarrow \infty}\left(\mathcal{J}_{t}^{\alpha} g, g\right)=0$ is a consequence of the monotone convergence theorem and the assumption that $g \in L^{2}(m)$. 
Theorem 2.2. $g \in \mathscr{D}\left(P_{T}^{-1}\right)$ if and only if

$$
\int_{0}^{\infty} I_{0}(2 \sqrt{2 T s})\left(\mathcal{J}_{s}^{\alpha} g, g\right) d s<\infty
$$

where $I_{0}$ is the modified Bessel function of the first kind of order 0. Moreover, if $g \in \mathscr{D}\left(P_{t}^{-1}\right)$, then $P_{t}^{-1} g$ equals a Bochner integral as follows:

$$
P_{T}^{-1} g=e^{-\alpha T} \int_{0}^{\infty} I_{0}(2 \sqrt{T s}) \mathcal{J}_{s}^{\alpha} g d s .
$$

Proof. It follows from (2.9) that

$$
\begin{aligned}
\int_{0}^{\infty} I_{0}(2 \sqrt{2 T s})\left(\mathcal{J}_{s}^{\alpha} g, g\right) d s & =\int_{0}^{\infty} d s I_{0}(2 \sqrt{2 T s}) \int_{0}^{\infty} \frac{1}{\lambda+\alpha} e^{-\frac{s}{\lambda+\alpha}} d\left(E_{\lambda} g, g\right) \\
& =\int_{0}^{\infty}\left(\int_{0}^{\infty} e^{-\frac{s}{\lambda+\alpha}} I_{0}(2 \sqrt{2 T s}) d s\right) \frac{1}{\lambda+\alpha} d\left(E_{\lambda} g, g\right) \\
& =\int_{0}^{\infty} e^{2 T(\lambda+\alpha)} d\left(E_{\lambda} g, g\right),
\end{aligned}
$$

which is finite if and only if $g \in \mathscr{D}\left(P_{T}^{-1}\right)$. The last line in the above follows from the Laplace transform of the modified Bessel function (see Table 29.3 in [1]).

Next observe that for $g \in L^{2}(m)$,

$$
\left\|\mathcal{J}_{s}^{\alpha} g\right\|^{2}=\int_{0}^{\infty} \frac{1}{(\lambda+\alpha)^{2}} e^{-\frac{2 s}{\lambda+\alpha}} d\left(E_{\lambda} g, g\right) \leq \frac{1}{e^{2} s^{2}} \int_{0}^{\infty} d\left(E_{\lambda} g, g\right)=\frac{\|g\|^{2}}{e^{2} s^{2}} .
$$

Thus, using Fubini's theorem and (2.9) we deduce

$$
\int_{0}^{\infty} I_{0}(2 \sqrt{T s}) \mathcal{J}_{s}^{\alpha} g d s=e^{\alpha t} \int_{0}^{\infty} e^{T \lambda} d E_{\lambda} g
$$

which implies (2.10).

$\mathcal{J}^{\alpha} g$ can be explicitly computed if one knows the transition function of $X$. If one instead has the knowledge of the family $\left(U^{\alpha}\right), \mathcal{J}^{\alpha} g$ is determined as the solution of a Cauchy problem.

Theorem 2.3. Given a $g \in L^{2}(m)$ there exists a unique solution to the following Cauchy problem:

$$
\begin{aligned}
\frac{d}{d t} j(t, \cdot) & =-U^{\alpha} j(t, \cdot) \\
j(0, \cdot) & =U^{\alpha} g .
\end{aligned}
$$

Moreover, its solution is given by $j(t, \cdot)=\mathcal{J}_{t}^{\alpha} g$.

Proof. Let $j(t, \cdot)=\mathcal{J}_{t}^{\alpha} g$ and observe using (2.9) that

$$
j(t, \cdot)=\int_{0}^{\infty} \frac{1}{\lambda+\alpha} e^{-\frac{t}{\lambda+\alpha}} d E_{\lambda} g .
$$


Thus,

$$
U^{\alpha} j(t, \cdot)=\int_{0}^{\infty} \frac{1}{(\lambda+\alpha)^{2}} e^{-\frac{t}{\lambda+\alpha}} d E_{\lambda} g .
$$

In view of the Fubini's theorem

$$
\int_{0}^{t} U^{\alpha} j(s, \cdot) d s=\int_{0}^{\infty} \frac{1}{\lambda+\alpha}\left(1-e^{-\frac{t}{\lambda+\alpha}}\right) d E_{\lambda} g=U^{\alpha} g-j(t, \cdot),
$$

which verifies that $\mathcal{J}_{t}^{\alpha} f$ solves (2.11) since

$$
\mathcal{J}_{0}^{\alpha} g=\int_{0}^{\infty} \frac{1}{\lambda+\alpha} d E_{\lambda} g=U^{\alpha} g .
$$

To show the uniqueness let us suppose $j_{1}$ and $j_{2}$ are two solutions of (2.11) and set $j=j_{1}-j_{2}$. Note that

$$
U^{\alpha} E_{\lambda} j(t, \cdot)=\int_{0}^{\lambda} \frac{1}{\mu+\alpha} d E_{\mu} j(t, \cdot) .
$$

Since $j$ solves (2.11) with the initial condition 0, applying $E_{\lambda}$ to both sides of the equality we obtain

$$
E_{\lambda} j(t, \cdot)=-\int_{0}^{t} d s \int_{0}^{\lambda} \frac{1}{\mu+\alpha} d E_{\mu} j(s, \cdot),
$$

which yields $E_{\lambda} j(t, \cdot)=0$ for all $\lambda \geq 0$. This completes the proof.

Since $-U^{\alpha}$ is a non-positive bounded operator, it generates a uniformly continuous semi-group, $T_{t}:=e^{-t U^{\alpha}}$. Thus, we have the following immediate corollary.

Corollary 2.3. Let $\left(T_{t}\right)$ be the semigroup on $L^{2}(m)$ generated by $U^{\alpha}$. Then,

$$
\mathcal{J}_{t}^{\alpha} g=T_{t} U^{\alpha} g \text {. }
$$

The fact that $\mathcal{J}_{t}^{\alpha} g$ is a solution of a Cauchy problem with a bounded generator also implies that one can compute it using a Picard iteration.

Corollary 2.4. Suppose $g \in L^{2}(m)$ and set

$$
\begin{aligned}
j_{0}(t, \cdot) & =U^{\alpha} g \\
j_{n+1}(t, \cdot) & =U^{\alpha} g-\int_{0}^{t} U^{\alpha} j_{n}(s, \cdot) d s .
\end{aligned}
$$

Then, $\left(j_{n}(\cdot, \cdot)\right)_{n \geq 0}$ converges uniformly in $L^{2}(m)$ to $\left(\mathcal{J}_{s}^{\alpha} g\right)_{s \in[0, t]}$ for any $t>0$, i.e.

$$
\lim _{n \rightarrow \infty} \sup _{0 \leq s \leq t}\left\|j_{n}(s, \cdot)-\mathcal{J}_{s}^{\alpha} g\right\|=0, \quad \forall t>0 .
$$

Proof. Let $j(s, \cdot)=\mathcal{J}_{s}^{\alpha} g$ and observe from (2.11) that

$$
j(s, \cdot)=U^{\alpha} g-\int_{0}^{s} U^{\alpha} j(r, \cdot) d r .
$$


Moreover,

$$
\begin{aligned}
\left\|j_{0}(s, \cdot)-j(s, \cdot)\right\| & \leq \int_{0}^{s}\left\|U^{\alpha} j(r, \cdot)\right\| d r=\int_{0}^{s} \sqrt{\int_{0}^{\infty} \frac{1}{(\lambda+\alpha)^{2}} e^{-\frac{2 r}{\lambda+\alpha}} d\left(E_{\lambda} g, g\right)} d r \\
& \leq \int_{0}^{s} \frac{e^{-\frac{r}{\alpha}}}{\alpha}\|g\| d r \leq\|g\| .
\end{aligned}
$$

Thus,

$$
\left\|j_{n+1}(s, \cdot)-j(s, \cdot)\right\| \leq \int_{0}^{s}\left\|U^{\alpha} j_{n}(r, \cdot)-U^{\alpha} j(r, \cdot)\right\| d r \leq \frac{1}{\alpha} \int_{0}^{s}\left\|j_{n}(r, \cdot)-j(r, \cdot)\right\| d r .
$$

Hence,

$$
\sup _{0 \leq s \leq t}\left\|j_{n+1}(s, \cdot)-j(s, \cdot)\right\| \leq \frac{1}{\alpha} \int_{0}^{t}\left\|j_{n}(r, \cdot)-j(r, \cdot)\right\| d r
$$

and we deduce by induction that

$$
\sup _{0 \leq s \leq t}\left\|j_{n}(s, \cdot)-j(s, \cdot)\right\| \leq \frac{t^{n}}{\alpha^{n} n !} \sup _{0 \leq s \leq t}\left\|j_{0}(s, \cdot)-j(s, \cdot)\right\| .
$$

In conjunction with (2.12) this leads to the estimate

$$
\sup _{0 \leq s \leq t}\left\|j_{n}(s, \cdot)-j(s, \cdot)\right\| \leq \frac{t^{n}}{\alpha^{n} n !}\|g\|,
$$

which yields the claim.

Although it is difficult to predict the tail behaviour of $\left(\mathcal{J}_{t}^{\alpha} f, f\right)$ as $t \rightarrow \infty$ due to the oscillatory nature of the Bessel functions of the first kind, the Laplace transform of $\left(\mathcal{J}_{t}^{\alpha} g, g\right)$ is a familiar object. Thus the tail behaviour can be determined by inverting this Laplace transform as well.

Proposition 2.2. Suppose $g \in L^{2}(m)$. Then for all $s \geq 0$

$$
\int_{0}^{\infty} e^{-s t}\left(\mathcal{J}_{t}^{\alpha} g, g\right) d t=\frac{1}{s} U^{\alpha+\frac{1}{s}}(g, g) .
$$

Proof. Using the spectral representation of $\mathcal{J}^{\alpha}$

$$
\begin{aligned}
\int_{0}^{\infty} e^{-s t}\left(\mathcal{J}_{t}^{\alpha} g, g\right) d t & =\int_{0}^{\infty} e^{-s t} \int_{0}^{\infty} \frac{1}{\lambda+\alpha} e^{-\frac{t}{\lambda+\alpha}} d\left(E_{\lambda} g, g\right) \\
& =\int_{0}^{\infty} \frac{1}{\lambda+\alpha} \frac{1}{s+\frac{1}{\lambda+\alpha}} d\left(E_{\lambda} g, g\right) \\
& =\frac{1}{s} \int_{0}^{\infty} \frac{1}{\lambda+\alpha+\frac{1}{s}} d\left(E_{\lambda} g, g\right)=\frac{1}{s} U^{\alpha+\frac{1}{s}}(g, g) .
\end{aligned}
$$

Also observe that the above identity is valid for $s=0$ since $\alpha U^{\alpha} g \rightarrow g$ as $\alpha \rightarrow \infty$ and

$$
\int_{0}^{\infty}\left(\mathcal{J}_{t}^{\alpha} g, g\right) d t=(g, g) .
$$


When $X$ is a one-dimensional transient diffusion we have yet another way of characterising $\mathcal{J}^{\alpha}$.

Proposition 2.3. Suppose that $X$ is as in Example 2.1 and is transient. Let $G_{x}:=$ $\sup \left\{t \geq 0: X_{t}=x\right\}$ be the last hitting time of $x$. Then

$$
\mathcal{J}_{t}^{\alpha} g(x)=u(x, x) E^{\mu}\left(J_{0}\left(2 \sqrt{t G_{x}}\right) e^{-\alpha G_{x}} \mathbf{1}_{\left[G_{x}>0\right]}\right),
$$

where $\mu$ is a measure on $(\mathbf{E}, \mathscr{E})$ given by $\mu(d y)=g(y) m(d y)$, and $u$ is the potential kernel for $X$, i.e.

$$
u(x, y)=\int_{0}^{\infty} p(t, x, y) d t
$$

Proof. It is well-known that (see, e.g., p.27 of [4])

$$
P^{y}\left(0<G_{y} \leq t\right)=\int_{0}^{t} \frac{p(s, x, y)}{u(y, y)} d s .
$$

In view of the symmetry of $p(t, x, y)$ the above implies for all bounded and continuous $h$ that

$$
E^{\mu} h\left(G_{x}\right) \mathbf{1}_{\left[G_{x}>0\right]}=\int_{0}^{\infty} h(s) \frac{P_{s} g(x)}{u(x, x)} d s,
$$

which yields the claim.

Recall (see Chapter 9 of [1]) that $J_{0}$ satisfies the following ODE:

$$
x^{2} J_{0}^{\prime \prime}+x J_{0}^{\prime}+x^{2} J_{0}=0 .
$$

The above equation and its connection with 2-dimensional Bessel process leads to the following remarkable observation that $\mathcal{J}_{t}^{\alpha}$ can be considered as the solution of a backward partial differential equation with an initial condition.

Proposition 2.4. Fix a $g \in L^{2}(m), T>0$ and consider the following function

$$
h(t, x)=\int_{0}^{\infty} J_{0}(2 \sqrt{x s}) e^{-2(T-t) s}\left(P_{s} g, g\right) d s, \quad x \geq 0, t \in[0, T) .
$$

Then

$$
\begin{aligned}
h_{t}+2 x h_{x x}+2 h_{x} & =0 ; \\
h(0, \cdot) & =\left(\mathcal{J}_{x}^{T} g, g\right) .
\end{aligned}
$$

Moreover, $\left(h\left(t, X_{t}\right)\right)_{t \in[0, S]}$ is a bounded martingale for any $S<T$ when $X$ is a 2dimensional squared Bessel process, i.e. $X$ is the unique weak solution

$$
d X_{t}=2 \sqrt{X_{t}} d W_{t}+2 d t
$$

where $W$ is a standard Brownian motion. 
Proof. First note that $\left|J_{0}\right|<1$ and $\frac{J_{0}^{\prime}(x)}{x}$ is bounded on $[0, \infty)$. The latter implies that $\frac{d}{d x} J_{0}(2 \sqrt{x s})$ is bounded whenever $s$ belongs to a bounded interval. In view of $(2.13)$ these observations further yield that $\frac{d^{2}}{d x^{2}} J_{0}(2 \sqrt{x s})$ is bounded when $(x, s)$ belong to compact squares. Thus, we can differentiate under the integral sign in (2.14) to get

$$
\begin{aligned}
& h_{t}+2 x h_{x x}+2 h_{x} \\
= & \int_{0}^{\infty}\left\{2 x \frac{d^{2}}{d x^{2}} J_{0}(2 \sqrt{x s})+2 \frac{d}{d x} J_{0}(2 \sqrt{x s})+2 s J_{0}(2 \sqrt{x s})\right\} e^{-2(T-t) s}\left(P_{s} g, g\right) d s .
\end{aligned}
$$

However, (2.13) implies that the term within the curly brackets vanishes. Moreover, $h(0, \cdot)=\left(\mathcal{J}_{x}^{T} g, g\right)$ by the definition of $h$. This completes the proof that $h$ solves the PDE in (2.15).

To finish the proof note that $\left(h\left(t, X_{t}\right)\right)_{t \in[0, S]}$ is a local martingale by an application of Ito's formula. Moreover, for any $t \leq S$

$$
0 \leq h\left(t, X_{t}\right) \leq \int_{0}^{\infty} e^{-2(T-S) s}\left(P_{s} g, g\right) d s=\left(U^{2(T-S)} g, g\right)<\infty,
$$

which in turn yields that $\left(h\left(t, X_{t}\right)\right)_{t \in[0, S]}$ is a bounded martingale.

\section{Regularisation of the inVERSE PROBlem}

Regularisation of inverse problems are in principle perturbations of the forward operator so that its inverse becomes a bounded operator on the underlying Hilbert space. As a bounded operator the perturbed inverse operator can then be applied to any member of the Hilbert space. If the perturbation is small, one expects not to deviate too much from the solution of the original inverse problem, if it exists. We refer the reader to [13] for an exhaustive treatment of regularisation methods for inverse problems.

The most common method for regularising ill-posed inverse problems is the Tikhonov regularisation. In our set up this will correspond to the solution of an auxiliary problem

$$
P_{T} f+\gamma f=g, \quad \gamma>0, g \in L^{2}(m) .
$$

Using spectral calculus it can be formally showed that the inverse of $P_{T}+\gamma I$ is given by

$$
\int_{0}^{\infty} \frac{1}{\gamma+e^{-\lambda T}} d E_{\lambda}
$$

Since $\gamma+e^{-\lambda T}$ is bounded away from 0 , this inverse operator is bounded and, therefore, has all of $L^{2}(m)$ as its domain.

In view of the above heuristic discussion we shall next describe a family of perturbations of the original problem that results in a regularisation. The resulting problems can be viewed as a mixture of the original inverse problem with a suitable regularising noise. 
Theorem 3.1. Suppose that $\phi: \mathbb{R}_{+} \rightarrow \mathbb{R}_{+}$is a continuous function with $\lim \inf _{x \rightarrow \infty} \phi(x)>$ 0 such that for some given $T>0$ and $\sup _{x \geq 0} e^{-T x} \phi(x)<\infty$. Then, for any $g \in L^{2}(m)$ there exists a unique solution $f \in L^{2}(m)$ to the following:

$$
(1-\gamma) P_{T} f+\gamma \phi(-A) f=g, \quad \gamma \in(0,1) .
$$

Moreover, the solution is given by

$$
f=\int_{0}^{\infty} \frac{1}{\gamma \phi(\lambda)+(1-\gamma) e^{-\lambda T}} d E_{\lambda} g
$$

and has the property that

$$
(1-\gamma) f=\underset{h \in L^{2}(m)}{\arg \min }\left\|P_{T} h-g\right\|^{2}+\frac{\gamma}{1-\gamma}\left(P_{T} \phi(-A) h, h\right) .
$$

Note that the choice of $\phi \equiv 1$ leads to the aforementioned Tikhonov regularisation.

Proof of Theorem 3.1 will follow from the following general result.

Lemma 3.1. Suppose that $B$ and $L$ are non-negative symmetric operators on a real Hilbert space $H$ with the inner product $(\cdot, \cdot)$ such that $B$ is bounded, the range of $B$ is included in $\mathscr{D}(L)$, and $B L=L B$. Then the following are equivalent for $f, g \in H$.

i) $f \in \mathscr{D}(L)$ and $B f+L f=g$.

ii) $f=\arg \min _{h \in H}\|B h-g\|+(B L h, h)$.

Proof. For $h \in H$ set

$$
J(h):=\|B h-g\|^{2}+(B L h, h)
$$

and observe that $B L h$ is well-defined since $B L=L B$ and the range of $B$ is included in $\mathscr{D}(L)$.

i) $\Longrightarrow$ ii) If i) holds, then $B^{2} f+B L f=B g$. Thus,

$$
\begin{aligned}
0 & =\left(B^{2} f-B g, f-h\right)+(B L f, f-h) \\
& =(B f-g, B f-B h)+(B L f, f)-(B L f, h) \\
& =(B f-g, B f-g)-(B f-g, B h-g)+(B L f, f)-(B L f, h) \\
& =J(f)-(B f-g, B h-g)-(B L f, h) \\
& =J(f)-J(h)+(B h-B f, B h-g)+(B L(h-f), h) .
\end{aligned}
$$

To arrive at ii) it remains to show $(B h-B f, B h-g)+(B L(h-f), h) \geq 0$ for any $h \in \mathscr{D}(L)$. Indeed, utilising $g=B f+L f$, we obtain

$$
\begin{aligned}
(B h-B f, B h-g)+(B L(h-f), h) & =\|B h-B f\|^{2}+(B L h, h)-2(B L f, h)+(B L f, f) \\
& =\|B h-B f\|^{2}+(B L(f-h), f-h),
\end{aligned}
$$

which is nonnegative since $B L$ is a nonnegative operator.

ii) $\Longrightarrow$ i) Since $f$ is the minimiser, $f \in \mathscr{D}(L)$ by hypothesis. Moreover, for any $h \in H$,

$$
\left.\frac{d}{d t} J(f+t h)\right|_{t=0}=0 \text {. }
$$


However, straightforward differentiation of $J(f+t h)$ yields

$$
\left(B^{2} f+B L f-B g, h\right)=0, \quad h \in H,
$$

which implies

$$
B^{2} f+B L f-B g=0 .
$$

On the other hand, $B^{2} f+B L f-B g$ is clearly in the domain of $B^{-1}$. Therefore, applying $B^{-1}$ to both sides of the above equation establishes i).

Proof of Theorem 3.1. Observe that $f$ given by (3.17) is well-defined and belongs to $L^{2}(m)$ since $g \in L^{2}(m)$ and $\liminf _{x \rightarrow \infty} \phi(x)>0$. Moreover, it belongs to the domain of $\phi(-A)$. The fact that $f$ is the solution of (3.16) is easy. Indeed, using the spectral representation

$$
(1-\gamma) P_{T} f+\gamma \phi(-A) f=\int_{0}^{\infty} \frac{e^{-\lambda T}(1-\gamma)+\gamma \phi(\lambda)}{\gamma \phi(\lambda)+(1-\gamma) e^{-\lambda T}} d E_{\lambda} g=g .
$$

Thus, it remains to show (3.18). However, this follows from Lemma 3.1 with $B=P_{t}$ and $L=\frac{\gamma}{1-\gamma} \phi(-A)$. Note that the range of $P_{T}$ is included in the domain of $\phi(-A)$ due to the assumption that $\sup _{x \geq 0} e^{-T x} \phi(x)<\infty$.

Remark 4. The choice of $\gamma$ in Theorem 3.1 is free and one can in fact obtain a statement independent of $\gamma$ by replacing $g$ by $(1-\gamma) g$ and $\phi$ by $\frac{\gamma}{1-\gamma} \phi$. However, the representation in (3.16) is chosen in order to emphasise that the regularisation can be viewed as a random mixture of two stochastic process. This point will be revisited and explained in more detail at the end of this section. Moreover, this representation will also be useful in Proposition 3.1 that studies the stability of the solutions of (3.16) as $\gamma \rightarrow 0$.

Remark 5. The assumption that $\liminf _{x \rightarrow \infty} \phi(x)>0$ cannot be dispensed easily if (3.16) is to have a solution for any given $g \in L^{2}(m)$. To see this take $\phi(x)=e^{-T x}$. Then (3.16) becomes $P_{T} f=g$, which does not have a solution in general.

If $g \in \mathcal{D}\left(P_{T}^{-1}\right)$, one should expect that the solutions of (3.16) converge to $P_{T}^{-1} g$ as $\gamma \rightarrow 0$. This is indeed the case as the following proposition shows.

Proposition 3.1. Let $\phi$ be as in Theorem 3.1 and for each $\gamma \in(0,1)$ denote by $f_{\gamma}$ the solution of (3.16). Assume further that $g \in \mathcal{D}\left(P_{T}^{-1}\right)$. Then

$$
\lim _{\gamma \rightarrow 0}\left\|f_{\gamma}-P_{T}^{-1} g\right\|=0 .
$$

Proof. The hypothesis that $g \in P_{T}^{-1}$ implies

$$
\int_{0}^{\infty} e^{2 \lambda T} d\left(E_{\lambda} g, g\right)<\infty .
$$


On the other hand,

$$
\left(e^{\lambda T}-\frac{1}{\gamma \phi(\lambda)+(1-\gamma) e^{-\lambda T}}\right)^{2} \leq\left(\frac{2-\gamma}{1-\gamma}\right)^{2} e^{2 \lambda T}
$$

Thus, in view of the Dominated Convergence Theorem, we have

$$
\lim _{\gamma \rightarrow 0} \int_{0}^{\infty}\left(e^{\lambda T}-\frac{1}{\gamma \phi(\lambda)+(1-\gamma) e^{-\lambda T}}\right)^{2} d\left(E_{\lambda} g, g\right)=0,
$$

which yields the claim.

Although looking abstract, Theorem 3.1 furnishes us with a plethora of concrete examples for regularising the inverse problem (1.1). To see this in a specific example suppose that the transition function, $\left(P_{t}\right)$, possesses a density with respect to $m$. Let us denote this transition density with $p(t, \cdot, \cdot)$ and introduce a new operator, $B$, on $L^{2}(m)$ via

$$
B f(x):=\int_{\mathbf{E}} f(y) p\left(T^{*}, x, y\right) m(d y)-f(x)=P_{T^{*}} f(x)-f(x),
$$

where $T^{*}>0$ is fixed. Due to the symmetry of $P_{T^{*}}, B$ is a also bounded symmetric operator on $L^{2}(m)$. Moreover, it corresponds to the generator of a Markov jump process that remains constant between the jumps of a Poisson process with unit parameter and moves between the points of $\mathbf{E}$ according to the transition function $P_{T^{*}}$ (see Example 2.2). Thus, by enlarging the probability space if necessary, we can assume the existence of a Markov jump process, $J$, with generator $B$ and independent from $X$. The semigroup, $\left(\tilde{P}_{t}\right)$, associated with $J$ is easily seen to satisfy $\tilde{P}_{t}=e^{t B}=$ $\phi(t ;-A)$, where $\phi(t ; x)=\exp \left(t\left(e^{-T^{*} x}-1\right)\right)$. Clearly, $\phi(T ; \cdot)$ satisfies the conditions of Theorem 3.1 for every $T>0$. Thus, for every $t>0, L^{2}(m)=\mathcal{D}\left(Q_{t}^{-1}\right)$, where

$$
Q_{t}=(1-\gamma) P_{t}+\gamma \tilde{P}_{t}
$$

Note that $\left(Q_{t}\right)$ is the semigroup of the Markov process, $Y$, where

$$
Y=\xi X+(1-\xi) J
$$

and $\xi$ is a Bernoulli random variable independent of $X$ and $J$ with $\operatorname{Prob}(\xi=1)=$ $1-\gamma$. Therefore, mixing the original Markov process with a pure jump process we observe that the inverse problem admits a solution. This construction readily extends to the following result.

Corollary 3.1. Suppose that $K$ is a bounded positive operator such that $K=\psi(-A)$ for some bounded continuous function $\psi: \mathbb{R}_{+} \rightarrow[0,1]$. In an enlargement of the probability space there exists a Markov process $Y$ such that

$$
Y=\xi X+(1-\xi) J
$$

where $\xi$ is a non-degenerate Bernoulli random variable, $J$ is a jump Markov process with generator

$$
B f=K f-f, \quad f \in L^{2}(m),
$$


and $\xi, J$ and $X$ are mutually independent. Moreover, $\mathcal{D}\left(Q_{t}^{-1}\right)=L^{2}(m)$, where $\left(Q_{t}\right)$ is the semigroup associated to $Y$.

Due to the relationship between the inverse problem and the backward PDEs the above corollary leads to the following in view of Corollary 2.2 .

Corollary 3.2. Suppose that $K$ is a bounded positive operator such that $K=\psi(-A)$ for some bounded continuous function $\psi: \mathbb{R}_{+} \rightarrow[0,1]$. Then, for any $g \in L^{2}(m)$ and $\gamma \in(0,1)$, there exists a unique solution of

$$
u_{t}+\gamma A u+(1-\gamma)(K u-u)=0, \quad u(0, \cdot)=g .
$$

The above corollaries show that if we construct a new process by randomly mixing the original process with a suitably chosen independent jump process, the inverse problem becomes well-posed when $P_{t}$ is replaced with the corresponding transition function of the new process. Note that $\gamma$ can be chosen arbitrarily close to 1 , which in practice means that one would almost never see the jump process, $Y$.

Moreover, the equation (3.20) provides a numerical algorithm to find the approximate solutions of the inverse problem when a sufficiently close approximation of $K$ is known as in the following examples.

Example 3.1. Suppose that $X$ is a Brownian motion and $m$ is the Lebesgue measure on the real line. Then, the generator $B$ defined in (3.19) corresponds to a compound Poisson process with unit intensity, whose jumps are normally distributed with mean 0 and variance $T^{*}$. In this case the process $Y$ of Corollary 3.1 is a Brownian motion with probability $1-\gamma$ and a compound Poisson process with probability $\gamma$.

Corollary 3.2, on the other hand, gives us a regularisation of the ill-posed backward heat equation with an initial condition. The regularisation takes the form of a partial integro-differential equation as follows:

$$
\begin{aligned}
u_{t}+\frac{\gamma}{2} u_{x x}+(1-\gamma) \int_{-\infty}^{\infty}(u(t, y)-u(t, x)) \frac{1}{\sqrt{2 \pi T^{*}}} \exp \left(-\frac{(x-y)^{2}}{2 T^{*}}\right) d y & =0 \\
u(0, \cdot) & =g .
\end{aligned}
$$

Corollary 3.2 yields the existence and uniqueness of a solution to the above for any $g \in L^{2}(m)$.

Example 3.2. Let $K=\alpha U^{\alpha}$ for some $\alpha>0$, where $U^{\alpha}$ is the $\alpha$-potential operator. Observe that $\|K\| \leq 1$ so Corollary 3.1 is applicable. In this case the process $Y$ is given by $X$ with probability $1-\gamma$ while it is equal to a Markov jump process with generator $K-I$ with probability $\gamma$.

Example 3.3. If the potential operator $U$ is bounded, then $K=\frac{U}{\|U\|}$ will have norm 1 to which one can apply Corollary 3.1. A particular example of this situation occurs when $X$ is a one-dimensional diffusion on $(\ell, r)$ with absorbing boundaries. In this case $U$ is bounded and it has the representation

$$
U f(x)=\int_{\ell}^{r} v(x, y) f(y) m(d y)
$$


where $m$ is the speed measure, $v(x, y)=s(x \wedge y)(1-s(x \vee y))$, and $s$ is the normalised scale function so that $s(\ell)=0$ and $s(r)=1$. Therefore, in view of Corollary 3.2, an approximate solution to the inverse problem is given by the solution of

$$
\begin{aligned}
u_{t}(t, x)+\gamma A u(t, x)+(1-\gamma) \int_{\ell}^{r}\{u(t, y)-u(t, x)\} \frac{v(x, y)}{\|U\|} m(d y) & =0 \\
u(0, \cdot) & =g .
\end{aligned}
$$

Remark 6. The above examples and more generally Corollary 3.2 show that the solution of the regularised inverse problem coincides with that of a partial integrodifferential equation. Using the approach of [8] one can obtain a finite difference scheme that approximates the solution. Development of an efficient numerical algorithm and its comparison with other regularisation methods is beyond the scope of this paper and left for future research.

\section{REFERENCES}

[1] Abramowitz, M. and Stegun,I.A. (1972): Handbook of mathematical functions: with formulas, graphs, and mathematical tables. National Bureau of Standards Applied Mathematics Series. Vol. 55. Corrected 10th printing.

[2] Back, K. (1992): Insider trading in continuous time. The Review of Financial Studies, 5(3), pp. 387-409.

[3] Bertero, M. and Boccacci, P. (1998): Introduction to inverse problems in imaging. CRC press.

[4] Borodin, A. N., and Salminen, P. (2012): Handbook of Brownian motion-facts and formulae, Birkhäuser.

[5] Campi, L., Cetin, U. and Danilova, A. (2011): Dynamic Markov bridges motivated by models of insider trading. Stochastic Processes and their Applications, 121(3), pp. 534-567.

[6] Cavalier, L. (2011): Inverse problems in statistics. Inverse problems and high-dimensional estimation, Lect. Notes Stat. Proc., 203, pp. 3-96.

[7] Chen, Z. Q. and Fukushima, M. (2012): Symmetric Markov processes, time change, and boundary theory, LMS-35 (Vol. 35), Princeton University Press.

[8] Cont, R. and Voltchkova, E. (2005): A finite difference scheme for option pricing in jump diffusion and exponential Lévy models. SIAM Journal on Numerical Analysis, 43(4), 15961626.

[9] Çetin, U. and Danilova, A. (2016): Markovian Nash equilibrium in financial markets with asymmetric information and related forward-backward systems. Ann. App. Prob., vol. 26, No. 4, pp. 1996-2029.

[10] Çetin, U. and Danilova, A. (2018): Dynamic Markov Bridges and Market Microstructure: Theory and Applications. Probability Theory and Stochastic Modelling (Vol. 90). Springer.

[11] Dunford, N. and Schwartz, J.T. (1971): Linear Operators. Part 3: Spectral Operators, WileyInterscience.

[12] Eggermont, P. P. B. and LaRiccia, V. N. (1995): Maximum smoothed likelihood density estimation for inverse problems. Ann. Statist., 23(1), pp. 199-220.

[13] Engl, H.W., Hanke, M. and Neubauer, A.. (1996). Regularization of inverse problems, Vol. 375, Springer Science \& Business Media.

[14] Elliott, J. (1955): Eigenfunction expansions associated with singular differential operators. Trans. Amer. Math. Soc., 78, pp. 406-425.

[15] Ethier, S., and Kurz, T. (1989): Convergence of Markov Processes, Wiley. 
[16] Falkner, N. (1983): Stopped distributions for Markov processes in duality. Zeitschrift für Wahrscheinlichkeitstheorie und Verwandte Gebiete, 62(1), pp. 43-51.

[17] Fukushima, M., Oshima, Y. and Takeda, M. (2010): Dirichlet forms and symmetric Markov processes. Studies in Mathematics, Vol. 19, de Gruyter.

[18] Ito, K., McKean, H. P., Jr. (1965): Diffusion processes and their sample paths, Springer.

[19] Koo, J-Y. and Chung, H-Y. (1998): Log-density estimation in linear inverse problems. Ann. Statist., 26(1), pp. 335-362.

[20] Kyle, A.S. (1985): Continuous auctions and insider trading. Econometrica, 53, pp. 1315-1335.

[21] McKean, Jr., H. P. (1956): Elementary solutions for certain parabolic partial differential equations. Trans. Amer. Math. Soc., 82, pp. 519-548.

[22] Shkolnikov, M., Sircar, R. and Zariphopoulou, T. (2016): Asymptotic Analysis of Forward performance processes in incomplete markets and their ill-posed HJB equations. SIAM J. Financial Mathematics, volume 7, pp. 588-618.

[23] Silverman, B. W., Jones, M. C., Nychka, D. W. and Wilson, J. D.. (1990): A smoothed EM approach to indirect estimation problems, with particular reference to stereology and emission tomography. J. Roy. Statist. Soc. Ser. B, 52(2), pp. 271-324.

[24] Vardi, Y., and Lee, D. (1993) From image deblurring to optimal investments: Maximum likelihood solutions for positive linear inverse problems. Journal of the Royal Statistical Society. Series B (Methodological), pp. 569-612.

[25] Widder, D. V. (1951): Necessary and sufficient conditions for the representation of a function by a Weierstrass transform. Trans. Amer. Math. Soc., 71, pp. 430-439.

Department of Statistics, London School of Economics and Political Science, 10 Houghton st, London, WC2A 2AE, UK

Email address: u.cetin@lse.ac.uk 